\title{
Necessidades Especiais de Escolares com Diabetes Mellitus Tipo 1 Identificadas Por Familiares ${ }^{1}$ \\ Special NeEds of Students With Diabetes Mellitus Type 1 Identified \\ BY RELATIVES
}

\author{
Tania Moron Saes BRAGA² \\ Diogo Pazzini BOMFIM ${ }^{3}$ \\ Daher SABBAG FILHO ${ }^{4}$
}

\begin{abstract}
RESUMO: É um desafio atender aos alunos, em suas diversas necessidades especiais. Diferentemente das deficiências, há poucas pesquisas no Brasil sobre estudantes com doenças crônicas e suas especificidades, na escola. O objetivo do presente estudo foi identificar as necessidades especiais de escolares com diabetes mellitus tipo 1. Participaram 37 familiares de escolares em tratamento ambulatorial de uma faculdade paulista, por meio de entrevista semiestruturada, gravada em áudio e transcrita para análise. Os resultados mostraram que todos comunicaram a escola a respeito da doença do filho, mas, mesmo assim, 29,7\% relatam dificuldade de inclusão ou acesso à escola, como desconhecimento do professor para o controle do diabetes, merenda escolar inadequada, preconceito dos colegas e da diretora ou vergonha por parte do aluno. As faltas ocorrem com 70,3\% dos alunos, principalmente devido às consultas médicas. Necessidades especiais foram identificadas por 32,4\%, incluindo a alimentação, o desempenho escolar e a necessidade de profissionais da escola mais bem informados sobre a doença. Além disso, 72,9\% referem algum tipo de apoio para enfrentar o diabetes, principalmente de profissionais de saúde. Dos familiares, 51,3\% apresentam sugestões para um melhor desenvolvimento do filho na escola, incluindo alimentação escolar adequada e melhor preparo da escola para lidar com o diabetes, como palestras e treinamento aos professores. Sugerimos a aproximação entre a escola e a área da saúde, porém, o primeiro passo para a integração intersetorial é identificar as necessidades desses alunos com doenças crônicas, como foi possível com este trabalho, ao enfocar o diabetes mellitus e suas repercussões escolares.
\end{abstract}

PALAVRAS-CHAVE: Educação Especial. Inclusão. Articulação saúde-educação. Desenvolvimento e aprendizagem.

ABSTRACT: Catering to students according to their various special needs is a challenge. As opposed to the field of disability, there is little research in Brazil on students with chronic diseases and their specific needs at school. The purpose of this study was to identify the special needs of children with diabetes mellitus type 1 . The participants were 37 family members of students in treatment in an outpatient center at a state of São Paulo medical school. A semi-structured interview was carried, which was audio recorded and transcribed for analysis. The results showed that all participants informed the school informed about their child's condition, however $29,7 \%$ reported that inclusion or access to school was difficult, due to the teacher being uninformed about diabetes control, inadequate school lunches, and preconceptions of peers and school supervisor, or the student's embarrassment. Absenteeism occurred among 70,3\% of the students, mainly due to doctor appointments. Special needs were identified by $32,4 \%$, including meals, performance in school tasks and the need to better inform school professionals about the condition. Furthermore, 72,9\% reported some kind of support to cope with diabetes had

\footnotetext{
${ }^{1}$ Financiamento: PIBIC/CNPq. Universidade Estadual Paulista “Júlio de Mesquita Filho"- Campus de Marília.

${ }^{2}$ Psicóloga. Doutora. Docente do Departamento de Educação Especial da Universidade Estadual Paulista (UNESP) Campus Marília. E-mail: tania.icm@icm.com.br

${ }^{3}$ Acadêmico de Medicina da Faculdade de Medicina de Marília (Famema). Bolsista PIBIC/CNPq em Educação Especial, UNESP Campus Marília. diogopazzini@famema.br

${ }^{4}$ Médico. Doutor. Docente da Disciplina de Pediatria da Famema. E-mail: dahersabbag@yahoo.com.br
} 
been provided, mainly by health professionals. In order to improve their child's development at school $51,3 \%$ presented suggestions such as including appropriate school meals, better preparation of school for dealing with diabetes, i.e. lectures and training for teachers. Our suggestion is that there should be a closer relationship between the school and health services, but the first step towards intersector integration is to identify the needs of students with chronic diseases, such as this study showed, by focusing on diabetes mellitus and its impact on the school.

KEYWORDS: Special Education. Inclusion. Health needs. Development and learning.

\section{INTRODUÇÃo}

As escolas enfrentam o grande desafio de assistir os alunos, em suas mais diversas necessidades especiais. Parte dessa diversidade de estudantes, particularmente aquela com deficiências, tem sido mais comumente lembrada e, algumas vezes, contemplada com adequações necessárias. Os estudos de pesquisadores brasileiros na área de Educação Especial, em geral, têm enfocado a abordagem da inclusão escolar do aluno com deficiência, englobando principalmente condições como síndrome de Down, paralisia cerebral, deficiência mental, distúrbios da linguagem e deficiência auditiva (MANZINI, 2011; DE VITTA; DE VITTA; MONTEIRO, 2010; FERRAZ; ARAÚJO; CARREIRO, 2010; TAKASE; CHUN, 2010; BARBOSA; MOREIRA, 2009; SCHEMBERG; GUARINELLO; SANTANA, 2009). Entretanto, considera-se que uma parcela de estudantes não tem recebido oportunidades educacionais adequadas, além de não ter suas especificidades de saúde respeitadas, no ambiente escolar. Trata-se daqueles com doenças crônicas e suas consequentes necessidades especiais.

Percebe-se, pela prática do professor, não ser infrequente a presença de alunos com problemas de saúde na sala de aula. Porém, nota-se uma dificuldade de se obter estimativa válida da prevalência das condições crônicas de saúde. Lee (2007), em revisão de literatura, conclui que a variabilidade nos registros de prevalência nas taxas de doença crônica na infância pode ser explicada pela considerável diversidade nos conceitos e operacionalizações utilizadas. O autor sugere a necessidade de um consenso internacional sobre uma definição conceitual de condição crônica de saúde. Embora a variação encontrada seja grande, em torno de $0,22 \%$ a $44 \%$, isso não impede que se busque compreender as condições das crianças com problemas de saúde.

Newacheck e Taylor (1992), em seu estudo sobre a prevalência de doenças e/ou condições crônicas em menores de 18 anos, nos Estados Unidos da América (EUA), encontraram mais comumente as alergias respiratórias (97 por 1000) e frequentes infecções de ouvido (83 por 1000). Outras doenças e/ou condições crônicas comuns foram a asma, eczema e alergias da pele, frequentes ou graves cefaleias e anomalias na fala. Com prevalência mais baixa, foram encontradas: diabetes, anemia falciforme e paralisia cerebral, cada uma afetando menos do que duas por mil crianças, nos EUA. 
Segundo dados de uma pesquisa nacional de saúde, realizada nos EUA entre 1992 e 1994, foi calculado que 4,4 milhões de crianças, ou 6,5\% dos menores de 18 anos, tinham uma condição crônica bastante severa, a ponto de interferir na participação em atividades diárias, como as escolares (NEWACHECK; HALFON, 1998), enquanto um milhão de crianças estava se tratando de uma doença crônica que limitava suas habilidades para frequentar a escola (THOMPSON Jr; GUSTAFSON, 1997).

Nesse sentido, pode-se deduzir que muitos educadores trabalham, ou certamente irão trabalhar, com estudantes que tenham alguma condição especial de saúde. Verifica-se, dessa forma, a importância de investigação com os objetivos de identificar necessidades específicas desses alunos, de seus familiares e dos profissionais envolvidos. Trata-se de um quadro cujo pano de fundo estampa a bem conhecida falta de preparo dos professores, a falta de apoio de outros profissionais da saúde, em particular do enfermeiro escolar, e de uma legislação pouco clara, ao tratar desses aspectos específicos e, portanto, com baixa aplicação na prática.

De modo geral, a doença crônica inclui a interação entre suscetibilidade biológica, tratamento e outros fatores do ambiente. Tais doenças podem ser manejadas no intuito de diminuir o sofrimento e melhorar a qualidade de vida dos doentes, porém, na maioria dos casos, não são completamente curadas. As hospitalizações prolongadas, principalmente no período de diagnóstico, são frequentes (BRADFORD, 1997; PERRIN; SHONKOFF, 2000). Na infância, as doenças e/ ou condições crônicas mais comuns são a asma, as desordens alérgicas, as desordens digestivas, as desordens do sistema nervoso central e os ataques como epilepsia (NEWACHECK; HALFON, 1998). O tratamento é caracterizado por deteriorações físicas que podem envolver muitos contatos com médicos, hospitalizações, procedimentos médicos invasivos, estresse e desconforto na criança e sua família (GARRALDA, 1994; WALLANDER; VARNI, 1998).

Em nossa realidade, Nonose (2009), usando chek list para o estudo de doenças crônicas entre os estudantes de um município localizado no Noroeste do Paraná, encontrou uma prevalência de $60 \%$ dos escolares com uma ou mais condições crônicas de saúde. Desse total, 34\% dos escolares evidenciam uma condição crônica, enquanto $26 \%$ apresentam mais de uma. Algumas doenças e/ ou condições crônicas mais prevalentes na literatura também foram encontradas nessa pesquisa. Dentre elas, estão: anemia, doenças pulmonares, amigdalites repetitivas, cefaleia, diabetes, doença cardíaca, epilepsia e otite crônica.

Além de dados de prevalência, pesquisas na área sobre outras informações também são escassas. De acordo com Shiu (2001), a quantidadelimitada de pesquisas direcionadas a investigar as necessidades de estudantes com doenças crônicas é paralela à escassa atenção dada na prestação de serviços. Os profissionais da saúde frequentemente lembram aos educadores a correlação entre saúde e desempenho acadêmico, mas essa correlação não é sempre acompanhada de discussão sobre os fatores e condições específicas, sendo raramente exposta com evidências. 
Conforme DePaepe, Garrison-Kane e Doelling (2002), o aumento de estudantes dessa categoria exige educadores mais responsivos às suas necessidades. Os autores sugerem conhecimentos e habilidades para educadores no atendimento aos alunos com deficiências de saúde, tais como: conhecimentos de condições de saúde e seus efeitos no desenvolvimento e comportamento do estudante; métodos de identificação das necessidades e planos para responder às necessidades especiais de saúde e educacionais, com adaptação e implementação de tecnologia assistida apropriada; e determinação de procedimentos para uso de auxílios e serviços suplementares.

Considerando que o conhecimento produzido sobre esse tema é insuficiente e sua importância para o planejamento e organização dos serviços de saúde e educação, visando à colaboração entre família e os profissionais da educação e saúde, este artigo enfocará uma doença crônica em especial: o diabetes mellitus (DM). Serão apresentados dados de um estudo realizado com familiares de escolares com DM, relativos às repercussões escolares e suas necessidades no ambiente da escola, pela percepção dos próprios familiares.

A atual classificação divide o DM em: DM tipo 1 (DM1), DM tipo 2 (DM2), outros tipos específicos de DM e DM gestacional. Apesar do aumento do DM2, na infância e na adolescência, nessa faixa etária ainda predomina o DM1. A etiopatogenia do DM1 resulta da destruição das células beta pancreáticas, mediada pela resposta autoimune celular, levando à falta de insulina. Já o DM2 é resultante de resistência periférica à insulina e/ou relativa deficiência de insulina (AMERICAN DIABETES ASSOCIATION, 2011).

Sabe-se que a incidência de DM tipo 1 vem crescendo, particularmente na população infantil com menos de cinco anos de idade. No Brasil a incidência do DM1 por 1000 habitantes é 7,6. Conceitualmente, o DM é um grupo de distúrbios metabólicos caracterizado pela hiperglicemia. Essa hiperglicemia é o resultado de defeitos na ação da insulina, na secreção de insulina ou em ambos (SOCIEDADE BRASILEIRA DE DIABETES, 2007).

Tendo em vista os cuidados regulares e constantes exigidos para o controle do DM e em decorrência das necessidades que podem surgir no ambiente escolar, este estudo tem como objetivo identificar tais necessidades junto aos familiares de escolares com DM1.

\section{MÉTodo}

Trata-se de uma pesquisa do tipo descritivo. $\mathrm{O}$ estudo foi realizado no Ambulatório de Pediatria da "nome da instituição", em Marília (SP). Nesse local, funcionam os "Grupos de Diabetes", onde pacientes e familiares, além do atendimento médico recebido, participam de palestras educativas sobre saúde e realizam dinâmicas com troca de experiências, elaboradas por uma equipe multiprofissional (médico, enfermeira, técnica de enfermagem, nutricionista, 
farmacêutico, psicóloga, alunos da graduação de Medicina e Enfermagem e, eventualmente, outros profissionais).

Participaram do estudo 37 familiares de crianças e adolescentes com diagnóstico de DM1 há pelo menos um ano, em acompanhamento nesse ambulatório, que fossem o principal cuidador e que desejassem participar. $\mathrm{O}$ período de um ano foi estabelecido para que o familiar tivesse se recuperado do impacto do diagnóstico e já estivesse vivenciando a experiência da doença. Os participantes assinaram o Termo de Consentimento Livre e Esclarecido. Houve aprovação do projeto pelo Comitê de Ética em Pesquisa da instituição local (protocolo $\mathrm{n}^{\mathrm{o}} \mathrm{x} x \mathrm{x} / \mathrm{xx}$ ). Todos os procedimentos foram realizados conforme a Resolução No 196/96 sobre pesquisa com seres humanos (BRASIL, 1996).

O instrumento utilizado na coleta de dados foi elaborado com base nos objetivos do estudo e na literatura, passou por etapas de adequação, com aplicação de entrevista-piloto em população semelhante, que não participou da pesquisa, e ajustes necessários, posteriormente. Os dados foram coletados por meio de entrevista semiestruturada, em local privativo do ambulatório, com duração média de meia hora, gravação em áudio e transcrição para análise. O período de realização das entrevistas foi de dezembro de 2008 a março de 2009, dando oportunidade de participar a todos os familiares que se enquadraram nos critérios. A entrevista é um instrumento que orienta uma conversa com finalidade e um facilitador de abertura, ampliação e aprofundamento da conversação (MINAYO, 2006).

As questões que nortearam a entrevista são do tipo: " $\mathrm{O}(\mathrm{A})$ senhor(a) teve alguma dificuldade na inclusão escolar do seu filho com diabetes?", "Em sua opinião, o seu filho tem alguma necessidade especial no ambiente escolar?" Outras questões foram acrescidas para compreender melhor as necessidades do aluno com DM1, pela percepção dos familiares.

A fim de se analisar os resultados qualitativos, buscou-se a análise do conteúdo, com o objetivo de alcançar um sentido estável, conferido pelo locutor no próprio ato de produção do texto (BARDIN, 2003). Para a análise de dados quantitativos dos participantes, empregou-se o programa Epi Info, versão 6.02 (PUBLIC HEALTH SERVICE, 1994).

\section{Resultados}

\section{1 CARACTERIZAÇÃo dos FAMiliaRes do ESTUdo}

Participaram do estudo 30 mães, cinco pais, uma avó materna e uma avó paterna, com idades variando de 28 a 67 anos, idade média de 41,6, desviopadrão $(\mathrm{DP})=+-9,2$. A maioria dos familiares é casada $(67,6 \%)$, predominando a religião católica $(56,8 \%)$. Os participantes são procedentes de diversas localidades, totalizando 15 municípios do interior do Estado de São Paulo. A escolaridade variou de analfabetismo até ensino superior completo, com predomínio de ensino fundamental incompleto (40,5\%). A maioria dos familiares é do lar, isto é, não 
trabalha fora de casa $(45,9 \%)$ e, em segundo lugar, aparecem as empregadas domésticas $(21,6 \%)$. A caracterização da amostra de familiares é apresentada na Tabela 1.

Tabela 1 - Caracterização dos familiares de crianças e adolescentes com diabetes mellitus tipo 1. Marília, 2009

\begin{tabular}{l|l|l|l|l|l}
\hline Familiar & $\mathbf{n}$ & $\%$ & Escolaridade & $\mathbf{n}$ & $\%$ \\
Mãe & 30 & 81,1 & Analfabetismo & 01 & 2,7 \\
Pai & 05 & 13,5 & Ensino Fundamental Incompleto & 15 & 40,5 \\
Avó materna & 01 & 2,7 & Ensino Fundamental Completo & 03 & 8,1 \\
Avó paterna & 01 & 2,7 & Ensino Médio Incompleto & 02 & 5,4 \\
Total & 37 & 100 & Ensino Médio Completo & 14 & 37,8 \\
Estado civil & $\mathbf{n}$ & $\%$ & Ensino Superior Completo & 02 & 5,4 \\
Casado (a) & 25 & 67,6 & Total & 37 & 100 \\
Viúvo (a) & 06 & 16,2 & Profissão & $\mathbf{n}$ & $\%$ \\
Divorciado(a) & 03 & 8,1 & Do lar & 17 & 45,9 \\
Solteiro(a) & 0,3 & 8,1 & Empregada doméstica & 08 & 21,6 \\
Total & 37 & 100 & Funcionários de indústria e comércio & 04 & 10,8 \\
Religião & $\mathbf{n}$ & $\%$ & Trabalhador(a) rural & 03 & 8,1 \\
Católica & 21 & 56,8 & Pedreiro & 02 & 5,4 \\
Evangélica & 12 & 32,4 & Costureira & 01 & 2,7 \\
Espírita & 02 & 5,4 & Funcionário(a) público & 01 & 2,7 \\
Agnóstica & 01 & 2,7 & Cuidadora de idosos & 01 & 2,7 \\
Nenhuma & 01 & 2,7 & Desempregado & 2,7 \\
Total & 37 & 100 & Total & 2,7 \\
\hline
\end{tabular}

A seguir, serão expostos dados que caracterizam os escolares com DM1, filhos dos participantes-alvo deste estudo, por se considerar que podem ser úteis na compreensão dos resultados.

Um total de 41 crianças/adolescentes participou do estudo, sendo 21 do sexo feminino e 20 do sexo masculino, cujas idades variaram de seis a 19 anos, idade média de 13,7, (DP = +-3,2). A escolaridade variou da $2^{a}$ série do ensino fundamental até $1^{\mathrm{a}}$ série do ensino superior, com predomínio de escolas públicas $(92,7 \%)$. O tempo de diagnóstico variou de um a 19 anos, com tempo médio de 8,7 anos $(\mathrm{DP}=+-4,4)$. 


\section{2 RELATO dos FAMILIARES SOBRE ASPECTOS RELACIONADOS AO ALUNO COM DM NA ESCOLA}

Visando a levantar informações do aluno na escola foram investigados os seguintes aspectos: a) comunicação do diagnostico à escola; b) o acesso à escola; c) o comparecimento às aulas; d) necessidades especiais identificadas; d) apoio recebido; e) sugestões.

A Tabela 2 reúne de forma resumida os dados quantitativos.

Tabela 2 - Frequência dos relatos dos familiares sobre aspectos relacionados ao aluno com diabetes mellitus tipo 1 na escola. Marília, 2009.

\begin{tabular}{lllllll}
\hline $\begin{array}{l}\text { Aspectos } \\
\text { relacionados } \\
\begin{array}{l}\text { ao aluno com } \\
\text { DM1 }\end{array}\end{array}$ & $\begin{array}{l}\text { Comunicaram } \\
\text { o diagnóstico } \\
\text { à escola }\end{array}$ & $\begin{array}{l}\text { Tiveram } \\
\text { dificul- } \\
\text { dade de } \\
\text { acesso }\end{array}$ & Faltas & $\begin{array}{l}\text { Identificam } \\
\text { necessida- } \\
\text { des espe- } \\
\text { ciais }\end{array}$ & $\begin{array}{l}\text { Relatam } \\
\text { apoio }\end{array}$ & $\begin{array}{l}\text { Fornecem } \\
\text { sugestões } \\
\text { para o } \\
\text { desenvol- } \\
\text { vimento do } \\
\text { filho }\end{array}$ \\
\hline $\mathbf{n}^{*}$ & 37 & 11 & 26 & 12 & 27 & 19 \\
\hline Porcentagem & $100 \%$ & $29,7 \%$ & $70,3 \%$ & $32,4 \%$ & $72,9 \%$ & $51,3 \%$ \\
\hline
\end{tabular}

$\mathrm{n}=$ número de familiares

a) Comunicação do diagnóstico: Todos os pais comunicaram a escola sobre a condição de saúde do filho; relatam que levam atestados e conversam com os diretores, no início do ano letivo. É um dado que não deixa dúvida quanto à notificação da escola do problema de saúde do escolar, no entanto, não há garantias, pelos relatos, sobre o conhecimento do professor no manejo ao aluno diabético.

b) Acesso: Buscou-se investigar se os pais tiveram dificuldade na inclusão do filho na escola. Na Tabela 2, pode ser visto que 11 familiares respondem afirmativamente. A análise qualitativa das respostas (Quadro 1) permite identificar que as barreiras relatadas são relacionadas: ao desconhecimento do professor para o controle da DM (4 relatos), merenda escolar inadequada (3), preconceito dos colegas e da diretora ou vergonha pela por parte do aluno (3), sintomas que fazem o aluno não ir à escola (1). São barreiras impostas pela "dinâmica da escola", como as contadas pelas F13, F5, F23, F29, F32, F33, que evidenciam o despreparo dos professores e a refeição escolar não adequada, além das barreiras relatadas por F14 e F15, que, a princípio, parecem ser inerentes ao aluno, mas que podem ser ou não decorrentes de dificuldades geradas pelas condições da escola. A discriminação pelos colegas e, até mesmo, por membros da escola, como a diretora, além da vergonha por ter DM1 aparecem nos relatos de F4, F14 e F35. 
$\mathrm{F}^{*} 4$ "Teve rejeição dos amigos, alguns amigos rejeitaram [...] Porque ela teve mudança de escola e os alunos maiores rejeitaram [...] Não só os alunos, como a diretora também."

F5 "[...] na parte dos lanches, porque eu tenho que comprar, a escola não dá."

F13 "[...] as professoras qualquer coisa que ela sentia já ligavam para eu ir buscar, elas não faziam nada que pudesse ajudar $[. . .]^{\prime \prime}$

F14 "Ela tem vergonha de explicar para os colegas."

F15 "Ela passa mal e não vai para a escola."

F23 “A única dificuldade era em relação à refeição, a escola não dá uma refeição para diabético. Acredito que a grande preocupação dos pais é o que o filho vai comer na merenda da escola. Geralmente, é tudo à base de açúcar."

F29 "No começo tive dificuldade por causa da alimentação, mas foi resolvido. O doutor exigia que a merenda fosse diferente, tivesse mais frutas. E eles mudaram [...] Inclusive tinha o lugar dele pra se alimentar[...] Mas isso que acontece com ele eu imagino que não acontece com os outros."

F30 “Às vezes, ele passava mal na escola, a escola não fazia nada, eles ligavam em casa direto, ou então a professora trazia de carro pra minha casa, entregar na minha mão pra eu cuidar [...] Daí eu fui explicando, falei que se abaixar muito era pra dar um copo de água com açúcar. Agora eles já dão."

F32 "No começo, sim, agora não. Já estão acostumados com ele. Antes, abaixava."

F33 "Elas [as professoras] não sabem socorrer."

F35 "Algumas crianças mexiam com ele. E ele tem vergonha de levar a comida dele de casa."

Quadro 1 - Relatos dos familiares de alunos com diabetes mellitus tipo 1 em relação à inclusão escolar. Marília, 2009.

Legenda: F = Familiar.

c) Falta às aulas: O absenteísmo foi relatado por 70,3\% dos familiares. Verificouse que a maioria refere a consulta médica de rotina como justificativa para a falta às aulas. Também foi mencionado o DM1 descompensado, como motivo para se ausentar e/ ou procurar o especialista. Vale mencionar como ilustração o relato a seguir:

F5 "Ela falta no dia de consulta ou quando vejo que ela não está muito bem, porque a escola é muito longe e não daria tempo de eu chegar lá se ela precisasse ir embora."

Um fator agravante é que, para uma grande parcela dos participantes (59.4 \%), o controle médico não é realizado em sua cidade de origem, havendo necessidade de deslocamento para a cidade do local do estudo, referência regional na área de saúde. Assim, certamente, em dia de consulta, o aluno perderá um dia todo de aula.

d) Necessidades especiais: Foram identificadas por $32,4 \%$ dos familiares, que referiram à necessidade de alimentação adequada (7 relatos), o apoio ao 
desempenho escolar (3) e de profissionais da escola mais bem informados sobre a doença (3). Como pode ser visto no Quadro 2, a alimentação é uma preocupação e foi citada por 7 mães. Apesar da importância para o controle do diabetes, a alimentação é mencionada por apenas uma das mães como um cuidado que é prestado pela escola, após a mesma ter feito orientações aos funcionários. Além da necessidade de melhor formação profissional, ressaltada por 3 participantes, cabe destacar necessidades educativas citadas por 3 familiares e, em particular, a F13, um caso específico de educação especial, bem como um responsável que sublinhou serem necessárias adaptações nas aulas de educação física (F32).

\section{Alimentação $(n=7)$ *}

F5 "Eles deveriam fornecer o lanche, mas só tem ela com esse caso na escola, então eles não se interessam." F11 "Na alimentação, às vezes quando faz arroz doce, por exemplo, eles já separam, porque ela não pode." F27 "Necessidade de alimentação adequada."

F30 "Merenda [...] porque se ele não puder comer, ele não come."

F31 "Alimentação. Por isso vou pedir hoje um atestado médico para levar na secretaria de educação, para que, por exemplo, o dia que der um suco, dar um suco light, ou o dia que der um iogurte, dar um iogurte light."

F32 "Na alimentação e mesmo na educação física. Eu orientei as professoras dele, a merendeira." F37 "Alimentação, que teria que ser mudada."

Aprendizagem escolar - dificuldade cognitiva e sensorial $(n=3)$

F17 "Eu acho que ele tem dificuldade na leitura. Eu sempre achei ele muito esperto. Mas, depois que eu descobri o diabetes, faz 5 anos, ele não rendeu mais como eu achei que ele ia render, ele tem uma dificuldade maior, ele pega, mas é mais lento."

F7 "Ela é um pouco assim [...] Lerda, atrasada."

F13 "[...] era a vista, que ela não enxergava de longe na lousa."

Profissionais da escola informados sobre a doença $(n=3)$

F24 "Precisa de uma pessoa que saiba identificar os sintomas e eu converso isso com a diretora, no começo do ano e ela passa para os professores, para que, quando abaixe o açúcar no sangue, alguém dê algum alimento, telefone $[. . .]^{\prime \prime}$

F16 "Ela se torna uma pessoa que precisa de mais cuidado, todos os professores têm que estar mais informados, porque qualquer coisa que acontecer tem que socorrer de imediato, não dá para esperar chegar em casa. Não que eu quero que ela seja diferente dos outros, tem que ser igual."

F28 "Eles deveriam saber mais, a diretora da escola não sabe muita coisa. A professora sabe um pouco, porque já deu aula em outra cidade e tinha um aluno com diabetes. E hipoglicemia não avisa, quando você vê, já deu. E eles nem se interessam."

Quadro 2 - Relatos dos familiares de alunos com diabetes mellitus tipo 1 em relação à percepção de necessidades especiais. Marília, 2009.

Legenda: $\mathrm{n}=$ número de familiares.

e) Apoio recebido: Entre os familiares, 27 expressaram que tiveram um apoio no enfrentamento do DM1, ao passo que 10 não reconheceram nenhuma fonte 
de apoio. $\mathrm{O}$ apoio mencionado veio da própria família, de profissionais de saúde/Grupo de Diabetes, de amigos e de Deus/religião. Alguns participantes mencionaram mais de uma fonte de apoio, ao mesmo tempo. A categoria de apoio predominante é de Profissionais da Saúde/Grupo de Diabetes, com 11 relatos, seguida da família (9 relatos).

f) Sugestões fornecidas pelos familiares para um melhor desenvolvimento do escolar: Quando investigados se tinham sugestões que pudessem contribuir, no ambiente escolar, 19 dos familiares se manifestaram, como pode ser observado na Tabela 3.

Tabela 3 - Distribuição dos familiares entrevistados, de acordo com as sugestões que poderiam contribuir para o desenvolvimento do(a) filho(a), com diabetes mellitus, na escola. Marília, 2009

\begin{tabular}{lll}
\hline Sugestões & n & Porcentagem \\
\hline Alimentação escolar adequada para o aluno com diabetes & 11 & $57,9 \%$
\end{tabular}

Melhor preparo da escola para lidar com o diabetes, como palestras de informações sobre a doença; treinamento para atender o aluno com sintomas de urgência; mais cuidado e atenção com o aluno.

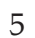

$26,2 \%$

Educação física diferenciada

Ajuda financeira do governo à família que tem um filho com diabetes

Mudança do horário de aula para períodos com temperatura mais amena

Total

$5,3 \%$

1 $5,3 \%$

1 $5,3 \%$

$19 \quad 100 \%$

\section{Discussões}

Os dados apresentados encontram apoio na literatura, o que legitima a família como fonte confiável de informações e parceira importante no cuidado de crianças e adolescentes com DM (THIES; McALLISTER, 2001; BROWN, 1999). Os resultados demonstram o indiscutível papel da família no cuidado ao aluno com DM e permitem reconhecer que muitos alunos com doenças ou condições crônicas de saúde podem ter dificuldades de aprendizagem, ainda não avaliadas, decorrentes de faltas às aulas, barreiras físicas e sociais, sintomas da doença, efeitos colaterais da medicação, entre outros. Uma vez mais, notamos, pela análise, 
a insuficiência na atuação dos profissionais da escola, que nos remete diretamente à formação. Não estamos afirmando com isso que esse profissional deverá ser formado para assumir mais tal cuidado, entretanto, precisa de informação, apoio e aproximação com os profissionais da saúde, para identificar os efetivos riscos e superar os desafios.

Partilhamos das propostas de Thies e McAllister (2001), de que as iniciativas de intervenção devem envolver família, escola e profissionais de saúde (médicos, enfermeiros e outros). Tais intervenções devem ser implementadas baseadas em nossa realidade, e recomendações sumárias, como sugeridas por DePaepe; Kane; Doelling (2002) poderiam ser seguidas pelos profissionais da educação. Na verdade, para Thies (1999), as crianças estão na intersecção dos dois sistemas: o da saúde o e da educação. O impacto da condição crônica da doença em suas habilidades para aprender não é discutido, porque, quando a criança está agudamente doente, o desempenho acadêmico não é percebido como prioridade e, quando essas crianças estão bem, a saúde não é percebida como um importante fator em sua educação.

Apesar de este estudo ter abordado o aluno com DM1, pode-se fazer um paralelo com as demais doenças crônicas, investigadas em nosso meio. Em amostra específica de pais de crianças com cardiopatia congênita, foi possível identificar que $90 \%$ afirmaram ter com os seus filhos condutas de superproteção. Outros fatores, como o baixo nível intelectual da mãe, comprometimentos de saúde (tuberculose, acidente vascular encefálico, dependência do álcool e drogas e depressão) e divergências entre os cônjuges sobre o tratamento foram fatores considerados de risco para o desenvolvimento e aprendizagem, podendo contribuir para deteriorar o desempenho das crianças com doenças cardíacas (BRAGA, 2004).

Investigação sobre escolares com otite média crônica, em entrevista com mães, demonstrou que as crianças adoeceram durante o período de aquisição da linguagem e que as mães tinham pouco conhecimento a respeito da doença, para fornecer estímulo adequado e assim evitar e superar os problemas decorrentes. Elas evidenciavam apreensão quanto às consequências da doença, como necessidade do uso de aparelhos de amplificação sonora e resultados de cura definitiva após cirurgia. Entre 10 mães que participaram do estudo, sete apontaram acentuada dificuldade no desempenho escolar dos filhos (CASTANHO; BRAGA, 2006). Da mesma forma, no atual estudo, três familiares referiram perceber dificuldade na aprendizagem escolar dos filhos.

Nonose (2009) fez um levantamento de necessidades de alunos com doenças e ou condições crônicas de saúde na escola, e 80\% dos familiares indicam necessidades dos filhos na escola, como a observação constante do filho para identificar sinais e sintomas de crises, alimentação adequada, adequação de atividades físicas, adequação de estrutura física, transporte escolar e participação de profissionais da saúde, bem como especialistas na escola. No atual estudo, essas necessidades são reforçadas, pois os familiares também destacam a necessidade de 
educadores que saibam atuar nas crises de hipoglicemia e hiperglicemia, e também de alimentação adequada, educação física compatível com as condições do aluno e aproximação da escola com profissionais de saúde.

Outro resultado relevante do atual estudo foi a questão das faltas escolares, que preocupa 70,3\% dos familiares da amostra. Em pesquisa de Shiu (2004), observou-se que as crianças cronicamente doentes apresentam riscos em decorrência de fatores como a ausência das aulas, que por si só aumenta a probabilidade de fracasso acadêmico, por dificultar o relacionamento entre os pares, que geralmente tendem a considerar o aluno faltoso como de fraco desempenho. Nesse contexto, a escola se torna um local propício para falhas acadêmicas e sociais. Nesse mesmo trabalho, foi proposto compreender as necessidades de pré-escolares com doenças crônicas, investigando junto aos pais e professores as necessidades e recomendações para apoio na escola. Os resultados indicam que pais de crianças com doença severa e moderada expressam como maior preocupação a falta às aulas, seguida do relacionamento com os colegas, o acesso, a habilidade dos profissionais da escola para reconhecer crises episódicas de saúde, o impacto no desempenho e a falta de conhecimento do professor sobre a doença, entre outros aspectos. Tais resultados são similares aos do nosso estudo.

É importante mencionar que as sociedades médicas especializadas fornecem diretrizes que reportam ao cenário da escola. De acordo com a Sociedade Brasileira de Diabetes (2006), são recomendados os seguintes cuidados na escola à criança e ao adolescente com DM: que os profissionais reconheçam os quadros de hipoglicemia, hiperglicemia e cetoacidose; a escola deve possuir um glicosímetro e saber utilizá-lo; permitir às crianças ir ao banheiro, alimentar-se ou tomar líquidos livremente, mesmo fora dos horários estabelecidos, e realizar monitorização glicêmica; possuir os telefones do serviço de saúde, médico e da família, caso sejam necessários; ter disponíveis insulina e glucagon, e pessoal treinado para aplicação em situações de emergência; e armazenar insulina em local adequado.

Todavia, há barreiras ao estudante com DM, como falta de acesso às ferramentas necessárias ao controle da doença (glicosímetro, insulina, comida/ líquidos, glucagon), falta de equipe na escola com conhecimento sobre DM e capacidade para prestar assistência, durante o período das atividades escolares (KAUFMAN, 2002). No Brasil, um estudo com 184 professores de educação infantil da cidade de Uberaba revelou que eles desconhecem as manifestações clínicas do DM $(27,7 \%)$, as abordagens terapêuticas $(33,7 \%)$ e as condutas diante de situações adversas (42,4\%), evidenciando a necessidade de capacitar os professores na temática DM, para abordar a criança com conhecimento e segurança (SIMÕES, 2010).

Portanto, um adequado número de profissionais da escola deveria ser treinado para executar os procedimentos necessários (isto é, monitorização da glicemia, administração de insulina e glucagon) e atuar nos momentos de hipoglicemia e hiperglicemia. Assim, estaria garantida, ao menos, a presença de um adulto capacitado durante as atividades escolares e mesmo nas extracurriculares, 
como passeios e viagens. Acredita-se que esses profissionais não precisam ser necessariamente da área da saúde, mas podem ser professores, administradores ou equipe de apoio, que tenham recebido formação em cuidados com diabetes, na escola (AMERICAN DIABETES ASSOCIATION, 2009).

Em investigação realizada por Jacquez et al. (2008), nos Estados Unidos da América, foi observado que os pais de crianças com DM têm preocupações em relação aos cuidados prestados pela escola, principalmente em situações de hipoglicemia e hiperglicemia, acreditando que ela não está capacitada para lidar com o DM. Além disso, a maioria das crianças não tinha permissão para verificar a glicemia e aplicar insulina em sala de aula.

Estudos internacionais da área afirmam que é preciso buscar formas de apoio a esses estudantes. Um caminho apontado por eles é o de se estabelecer relações de colaboração entre os educadores, os médicos e demais profissionais da saúde com a família (SHAW et al., 2004). De acord o com os autores, essa colaboração deve gerar diretrizes de apoio aos alunos, para vencer a situação adversa existente entre os educadores e os profissionais da saúde, um cenário em que não há delimitações claras para as demandas de fornecimento de educação especial ou serviços médicos e terapêuticos. Existe uma situação vista em que, por um lado, há médicos desrespeitosos ou ignorantes das políticas e da legislação escolar e, por outro, médicos que veem os profissionais da escola como fornecedores de informações irrelevantes das questões médicas de seu aluno, geradas por pedidos de prescrições preenchidos em formulários com jargões legais e educacionais por professores ou diretores. Os autores alertam que, se essa visão ocorre, mesmo que seja em uma parcela mínima, já afetaria a relação de colaboração, que, por sua vez, já sofre por barreiras sistemáticas, como o uso de sistemas diferentes de diagnóstico e intervenção. Frente a isso, os autores sugerem um modelo para desenvolver a colaboração entre médicos e educadores, o qual inclui diversos estágios, cuja base está no respeito mútuo e na comunicação clara, assim melhorando a qualidade de cuidado ao escolar.

Entendemos que o cuidado às crianças e adolescentes com DM, na escola, merece ser mediado por uma equipe. É necessária uma integração entre a família, profissionais de saúde e equipe escolar para dar proteção à criança durante esse longo período do dia em que está fora de casa. Com uma boa compreensão das condições do aluno com DM, dos seus cuidados de saúde e de suas necessidades acadêmicas, é possível planejar estratégias para contornar situações que possam inibir o potencial educacional do aluno ou comprometer sua saúde.

Essa sugestão para que os profissionais da escola trabalhem em equipe, no controle do DM, é defendida por autores como Getch, Bhukhanwala e NeuharthPritchettw (2007), os quais indicam estratégias para um plano individualizado. Esses mesmos autores concluíram, anteriormente, que poucos professores tinham treinamento formal para assistir crianças com condições médicas crônicas (NEUHARTH-PRITCHETT; GETCH, 2001). 


\section{Conclusões}

O presente estudo permitiu que a família identificasse necessidades especiais de saúde dos escolares com DM1, as quais podem ser supridas com planos de cuidados e pequenas adaptações na escola, ao lado de outras dependentes de ações políticas mais amplas, como a formação de professores e a aproximação entre educação e saúde. As doenças crônicas na infância, em especial o DM1, abordado no estudo, acarretam uma série de dificuldades, que incluem dificuldades de aprendizagem e uma quantidade considerável de especificidades no cotidiano escolar do aluno. As dificuldades de aprendizagem se referem a desempenhos escolares insatisfatórios descritos pelos familiares como "alunos com dificuldade na leitura", "atrasado", "aprende, mas é lento, lerdo e atrasado" e incluem, ainda, um aluno com necessidades educativas especiais associada à deficiência visual decorrente do diabetes. Foram lembrados os sintomas que afetam a participação nas atividades e os dias de aulas perdidos que podem ter um impacto no desempenho escolar. As condições do cotidiano escolar foram consideradas elementos importantes e que afetam o controle da doença como: merenda escolar inadequada; falta de informação dos profissionais da escola para eliminar o preconceito, e dos professores, em particular, para reconhecer sinais e sintomas necessários para o controle da doença. Ao dar voz aos familiares para falar de suas experiências e seus sentimentos, confirmou-se a valiosa fonte de informações que representam.

Sugerimos a aproximação entre escola e saúde. Esse elo, ao menos, pode ser realizado por meio de relatórios médicos, orientações escritas, participação de professores no Grupo de Diabetes e de profissionais da saúde, no ambiente escolar, realizando palestras educativas. Apesar de não serem ainda uma política específica e elaborada de integração, essas adaptações, talvez, já tragam benefícios ao aluno.

Porém, antes de tudo, é fundamental identificar e compreender as necessidades dos escolares com DM1 ou com qualquer outra doença crônica, como foi realizado nesta pesquisa. Este é o primeiro passo para se pensar em algum planejamento intersetorial. Um passo adicional foi dado a partir do levantamento de dados realizado neste estudo. Trata-se da elaboração de uma cartilha informativa para os professores visando atender às necessidades do aluno com diabetes, que já está sendo veiculada em diferentes meios de comunicação (BOMFIM et al., 2011).

Nesse sentido, entendemos ser necessária a continuidade da investigação, reflexão e possíveis planejamentos a respeito desse tema.

\section{REFERÊNCIAS}

AMERICAN DIABETES ASSOCIATION. Care of children with diabetes in the school and day care setting. Diabetes Care, Alexandria, v. 32, suppl, S68-S72, 2009. 
AMERICAN DIABETES ASSOCIATION. Standards of medical care in diabetes. Diabetes Care, v. 34, suppl 1, p. 11-61, 2011.

BARBOSA, A.J.G.; MOREIRA, P.S. Deficiência mental e inclusão escolar: produção científica em educação e psicologia. Revista Brasileira de Educação Especial, Marília, v. 15, n. 2, p. 337-352, 2009.

BARDIN, L. Análise de conteúdo. Lisboa: Edições 70, 2003.

BOMFIM, D. P. et al. O aluno com diabetes: Como contribuir para atender às necessidades do aluno com diabetes. ANAD, Marília, Nov. 2011. Disponível em: http:/ / www.anad.org.br/Diabetes/images/O_ALUNO_COM_DIABETES.pdf Acesso em: 12 nov. 2011.

BRAGA, T.M.S. Contribuições da análise do comportamento para a intervenção precoce em crianças com deficiências cardíacas e visuais. In: BRANDÃO, M.Z.S. et al. Sobre o Comportamento e Cognição: estendendo a psicologia comportamental e cognitiva aos contextos da saúde, das organizações da relações pais filhos e das escolas. 1. ed. Santo André: ESETec editores associados, 2004.

BRASIL. Ministério da Saúde. Conselho Nacional de Saúde. Normas de pesquisa envolvendo seres humanos - Resolução CNS 196/96. Bioética, 1996.

BROWN, R.T.; DUPAUL, G.J. Introdution to the mini-series : promoting school success in children with chronic medical conditions. School Psychology Review, v.28, n.2, p. 175-181, 1999.

CASTANHO, R.M., BRAGA; T.M.S. Indicadores do desempenho escolar em crianças com otite média crônica. Temas sobre Desenvolvimento. São Paulo, v. 15, p. 38 - 44, 2006.

DePAEPE, P.; GARRISON-KANE, L.; DOELLING, J. Supporting students with health needs in schools: an overview of selected health conditions. Focus on exceptional children, Denver, v.35, n.1, 2002.

DE VITTA, F. G. F.; DE VITTA, A; MONTEIRO, A. S. R. Percepção de professores de educação infantil sobre a inclusão da criança com deficiência. Revista Brasileira de Educação Especial, Marília, v.16, n.3, p. 415-428, 2010.

FERRAZ, C.R.A.; ARAÚJO, M.V.; CARREIRO, L.R.R. Inclusão de crianças com síndrome de Down e paralisia cerebral no ensino fundamental I: comparação dos relatos de mães e professores. Revista Brasileira de Educação Especial, Marília, v.16, n.3, p. 397-414, 2010.

GARRALDA, M. E. Chronic physical illness and emotional disorder in childhood. British Journal of Psychiatry, London, n.164, p. 8-10, 1994.

GETCH, Y.Q.; BHUKHANWALA, F.; NEUHARTH- PRITCHETT, S. Strategies for Helping Children With Diabetes in Elementary and Middle. Teaching Exceptional Children, Clemson, v.39, n.3, p.46-51, 2007.

JACQUEZ, F. et al. Parent perspectives of diabetes management in schools. The Diabetes Educator, Thousand Oaks, v. 34, n. 6, p. 996-1003, 2008.

KAUFMAN, F.R. Diabetes at school: what a child's health care team needs to know about federal disability law. Clinical Diabetes. Alexandria, v.20, n.2, p. 91-92, 2002. 
LEE, J.H. et al. Definitions and measurement of chronic health conditions in childhood. JAMA, Chicago, v.297, n.24, p.2741-2750, 2007.

MANZINI, E.J. Tipo de conhecimento sobre inclusão produzido pelas pesquisas. Revista Brasileira de Educação Especial, Marília, v.17, n.1, p.53-70, 2011.

MINAYO, M.C.S. O desafio do conhecimento: pesquisa qualitativa em saúde. São Paulo: Hucitec, 2006.

NEUHARTH-PRITCHETT, S.; GETCH, Y.Q. Asthma and the school teacher: The status of teacher preparedness and training. Journal of School Nursing, Thousand Oaks, v.17, p.323328, 2001.

NEWACHEK, P.W.; TAYLOR, W.R. Childhood chronic illness: prevalence, severity and impact. American Journal of Public Health, New York, v.82, n.3, p.364-371, 1992.

NEWACHECK, P.W.; HALFON, N. Prevalence and impact of disabling chronic conditions in childhood. American Journal of Public Health, New York, v.88, n.4, p.610-617, 1998.

NONOSE, E.R.S. Doenças crônicas na escola: um estudo das necessidades dos alunos. 2009. 98f. Dissertação (Mestrado em Educação) - Faculdade de Filosofia e Ciências, UNESP, Marília, 2009.

PERRIN, J.M.; SHONKOFF, J.P. Developmental disabilities and chronic illness: an overview. In: BEHRMAN, R.E.; KLIEGNAN, R.M.; JENSON, H.B. (Org.). Nelson textbook of Pediatrics. 16 ed. Filadelfia: W. B. Saunders, 2000.

PUBLIC HEALTH SERVICES. Department of Health and Human Services (US). Epi Info. [CD-ROM]. Versão 6.2. Atlanta: Centers for Disease Control and Prevention, 1994.

SCHEMBERG, S.; GUARINELLO, A.C.; SANTANA, A.P. As práticas de letramento na escola e na família no contexto da surdez: reflexões a partir do discurso dos pais e professores. Revista Brasileira de Educação Especial, Marília, v.15, n.2, p.251-268, 2009.

SHAW, et al. Collaborating with physicians: a guide for school leaders. Principal Leadership, Reston, v.5; p.11-13, 2004.

SHIU, S. Issues in the education of students with chronic illness. International Journal of Disability, Development and Education, London, v.48, n. 3, p. 269-281, 2001.

SHIU, S. Positive interventions for children with chronic illness: Parents' and teachers' concerns and recommendations. Australian Journal of Education, Camberwell, v. 48, n. 3, p. 239-252, 2004.

SIMÕES, A. L. A. Conhecimento dos professores sobre o manejo da criança com diabetes mellitus. Texto \& Contexto Enfermagem, Florianópolis, v.19, p.651-657, 2010.

SOCIEDADE BRASILEIRA DE DIABETES. Atualização brasileira sobre diabetes. Rio de Janeiro: Diagraphic, 2006. 142 p. Disponível em: <http:/ / dtr2004.saude.gov.br/dab/ docs/publicacoes/geral/atualizacao_diabetes2006.pdf>. Acesso em: 20 set. 2008.

SOCIEDADE BRASILEIRA DE DIABETES. Tratamento e acompanhamento do Diabetes mellitus. Diretrizes da Sociedade Brasileira de Diabetes, 2007. [citado 2011 mar 12] Disponível em: http:/ / www.anad.org.br/profissionais/images/Diretrizes_SBD_2007. pdf Acesso em 12 mar. 2011. 
TAKASE, E.M.; CHUN, R.Y.S. Comunicação e inclusão de crianças com alterações de linguagem de origem neurológica na perspectiva de pais e educadores. Revista Brasileira de Educação Especial, Marília, v. 16, n. 2, p. 251-264, 2011.

THIES, K.M. Identifying the educational implications of chronic illness in school children. Journal of school health, Kent, v. 69, n. 10, p. 392-397, Dec.1999.

THIES, K.M.; McALLISTER, J.W. The health and education leadership project: a school initiative for children and adolescents with chronic conditions. Journal of School Health, Kent, v.71, n.5, p.167- 172, 2001.

THOMPSON, R. J. Jr.; GUSTAFSON, K.E. Adaptation to chronic childhood illness. American Psychological Association, Whasington: 1997.

WALLANDER, J. L.; VARNI, J.W. Effects of pediatric chronical physical disorders on child and family adjustment. Journal of Pediatric Psychology, Atlanta, v.39, n.1, p.29-46, 1998.

Recebido em: 01/09/2011

Reformulado em: 21/05/2012

Aprovado em: 15/08/2012 
BRAGA, T. M. S.; BOMFIM, D. P.; SABBAG FILHO, D. 